\title{
The radiological interpretation of possible microbleeds after moderate or severe traumatic brain injury: a longitudinal study
}

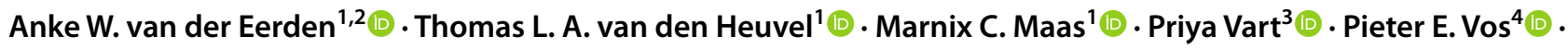

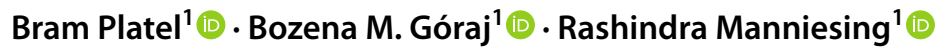

Received: 5 July 2021 / Accepted: 15 October 2021 / Published online: 31 October 2021

(c) The Author(s) 2021

\begin{abstract}
Introduction In order to augment the certainty of the radiological interpretation of "possible microbleeds" after traumatic brain injury (TBI), we assessed their longitudinal evolution on 3-T SWI in patients with moderate/severe TBI.

Methods Standardized 3-T SWI and T1-weighted imaging were obtained 3 and 26 weeks after TBI in 31 patients. Their microbleeds were computer-aided detected and classified by a neuroradiologist as no, possible, or definite at baseline and follow-up, separately (single-scan evaluation). Thereafter, the classifications were re-evaluated after comparison between the time-points (post-comparison evaluation). We selected the possible microbleeds at baseline at single-scan evaluation and recorded their post-comparison classification at follow-up.

Results Of the 1038 microbleeds at baseline, 173 were possible microbleeds. Of these, $53.8 \%$ corresponded to no microbleed at follow-up. At follow-up, 30.6\% were possible and $15.6 \%$ were definite. Of the 120 differences between baseline and follow-up, $10 \%$ showed evidence of a pathophysiological change over time. Proximity to extra-axial injury and proximity to definite microbleeds were independently predictive of becoming a definite microbleed at follow-up. The reclassification level differed between anatomical locations.

Conclusions Our findings support disregarding possible microbleeds in the absence of clinical consequences. In selected cases, however, a follow-up SWI-scan could be considered to exclude evolution into a definite microbleed.
\end{abstract}

Keywords Brain injuries $\cdot$ Traumatic $\cdot$ Cerebral hemorrhage $\cdot$ Traumatic $\cdot$ Diffuse axonal injury, $\cdot$ Magnetic resonance imaging $\cdot$ Longitudinal studies

\section{Introduction}

The yearly incidence of traumatic brain injury (TBI) is around 300 per 100,000 persons $[1,2]$. One of the injury types encountered in these patients is cerebral microbleeds (CMBs) [3-7]. Traumatic CMBs represent accumulations

Anke W. van der Eerden

a.vandereerden@erasmusmc.nl

1 Department of Radiology and Nuclear Medicine, Radboud University Medical Center, Nijmegen, Netherlands

2 Department of Radiology \& Nuclear Medicine, NE-515, Erasmus Medical Center, P.O. Box 2040, 3000 CA Rotterdam, Netherlands

3 Department of Epidemiology and Biostatistics, Radboud University Nijmegen, Nijmegen, Netherlands

4 Department of Neurology, Santiz-Slingeland Hospital, Doetinchem, Netherlands of hemosiderin-containing macrophages, usually caused by traumatic vascular injury [3]. They are associated with traumatic axonal injury $[8,9]$. Depending on their distribution, both are negatively associated with prognosis [3, 10-12].

The Microbleed Anatomical Rating Scale (MARS) [13] classifies microbleeds as "definite" or "possible" microbleeds within pre-defined anatomical brain-regions, with a high interobserver agreement for the assessment of definite microbleeds $[4,13,14]$. The assessment of possible microbleeds with GRE T2*-weighted 1.5-T MRI demonstrates substantial observer variability, due to equivocality in their interpretation as a CMB versus an alternative source of focal low signal intensity $[13,14]$. Therefore, to improve the reliability of microbleed rating, it is suggested to regard these possible microbleeds as "no finding" in a research setting [13]. In radiological practice, however, these equivocal findings are encountered daily, and the decision on how to interpret them in an individual patient is less straightforward. 
Indeed, the authors of the Brain Observer MicroBleed Scale paper suggested the performance of a longitudinal study to determine if possible microbleeds mature into definite CMBS (named "uncertain" and "certain" in their paper) [14].

In an attempt to augment the certainty of the radiological interpretation of possible microbleeds in daily practice, the purpose of this study was to assess the longitudinal evolution of possible microbleeds on SWI in patients with moderate or severe TBI. We also evaluated possible predictors of this evolution and assessed the causes of differences.

\section{Methods}

The data were obtained within a prospective observational long-term follow-up cohort study in consecutive patients with moderate or severe TBI (defined as head trauma resulting in Glasgow Come Scale score (GCS) 9-12 and 3-8 at the injury site, respectively), approved by Radboud University Medical Center's Institutional Review Board. The study was performed in accordance with the ethical standards as laid down in the 1964 Declaration of Helsinki and its later amendments. All patients or their next of kin gave written informed consent. This is the second publication on this study population [9].

\section{Study design}

Figure 1 summarizes the study design comparing SWI-findings obtained median $3(2-5(t 1))$ and $26(25-28(t 2))$ weeks after TBI. It defines the terms single-scan findings, postcomparison findings, and cause of difference used throughout the paper. We assessed how frequently possible microbleeds detected at $t l$ in the single-scan stage, were classified as no, possible, and definite microbleed at $t 2$ in the postcomparison stage. The single-scan findings at $t 1$ approach daily radiological practice: in a radiological setting, the decision on the interpretation of a possible microbleed needs to be made without any information on the findings at followup. Lacking correlation with pathological specimens, the post-comparison findings give information on the pathophysiological evolution: in the post-comparison stage the effects of interpretational uncertainty are minimized.

\section{Definitions}

We defined definite microbleeds as small, well-defined areas of SWI-signal void at least half surrounded by brain parenchyma, with clear margins ranging from 2 to $10 \mathrm{~mm}$ in size in at least one plane, with distinct appearance from potential mimics (e.g., calcium and iron deposits, bone, or vessel flow voids), which were not obviously part of an intraparenchymal hemorrhage or contusion. Possible microbleeds were less well defined or less hypointense (Table 1). Due to the lack of consensus on the definition of a traumatic CMB, this definition is an adaptation from non-traumatic literature (the STRIVE criteria [15], the Greenberg recommendations [5], and the MARS guidelines [13]), ignoring their criterion of sphericalness or ovalness, as traumatic CMBs can notably be elongated in shape $[6,16]$, and evaluating SWI instead of GRE T2*-weighted images. We use the term "microbleed" in a methodological sense. It comprises any finding classified as possible or definite microbleed at $t 1$ and/or at $t 2$. The term "CMB" is reserved for cerebral microbleed in a pathophysiological sense. The term "extra-axial injury score" is defined in the Online Resource.

\section{Patients and image acquisition}

The selection procedure described before [9] was prospectively applied to all 211 consecutive $\geq 18$-year-old patients having sustained a moderate or severe TBI $<24 \mathrm{~h}$ before presenting at the emergency department of Radboud University Medical Center, a level I trauma center. Finally, this yielded 31 patients with SWI and T1-weighted imaging data, obtained 3 (2-5) and $26(25-28)$ weeks after TBI on a single 3-T MRI scanner (Magnetom Trio, Siemens Healthineers) using the imaging parameters summarized in Table 2. The demographic and clinical patient characteristics were reported before [9], and they had a total of 865 definite and 173 possible microbleeds distributed over the patients and over the brain as summarized in Table 3 .

\section{Concomitant injury}

Information on concomitant injury, i.e., traumatic injury other than microbleeds, was collected at $t l$ as described in the Online Resource.

\section{MARS atlas}

In order to localize each microbleed or concomitant injury, we manually segmented the standard brain in Montreal Neurological Institute space [17] into the regions specified in the MARS scoring template [13]. The segmentation procedure was described previously [9]. We compounded putamen, globus pallidus, and nucleus caudatus into the region "basal ganglia."

\section{Microbleed evaluation}

Figure 1 summarizes the microbleed evaluation process. The Online Resource describes further details. 
Fig. 1 Overview of study procedure. $T B I$ traumatic brain injury, t1 3 (2-5) weeks after TBI, t2 26 (25-28) weeks after TBI,

$C A D$ computer-aided detection

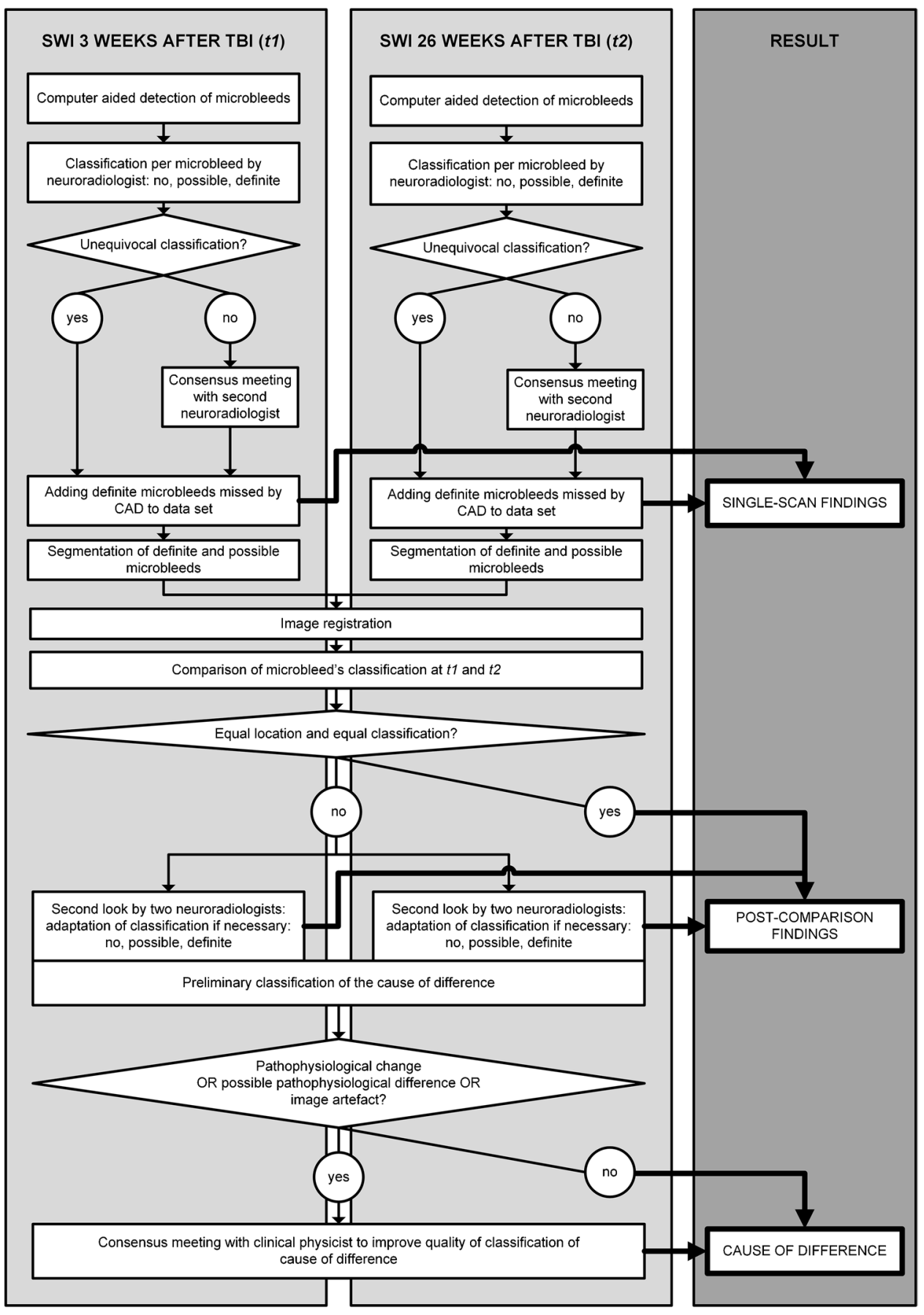

\section{Single-scan findings}

A computer-aided detection (CAD) system, described previously [18], evaluated the scans obtained at $t l$ and $t 2$, separately. The CAD system was set up with a high sensitivity, such that the chances of missing any possible microbleed was very low [18]. Blinded for the other timepoint, AE (neuroradiologist, 7 years of experience) classified each CAD-proposed microbleed as no, possible, or definite microbleed, deciding on CAD detections with the slightest doubt in consensus meetings with BG (neuroradiologist, 33 years of experience). Through visual screening, AE manually added definite microbleeds missed by the CAD system to the data set. Then, each definite or possible microbleed was automatically segmented using intensity-based volume-constrained region growing. Each microbleed was allocated to a MARS-region using the nonlinear registration tool FNIRT [19, 20]. In the rest of the paper, when referring to single-scan findings, we refer to our classification at this stage. 
Table 1 Definition of possible and definite microbleed

\begin{tabular}{ll}
\hline Definite microbleed & Possible microbleed \\
\hline $\begin{array}{l}\text { Area of SWI signal void } \\
\text { At least half surrounded by brain parenchyma }\end{array}$ & May be less hypo-intense \\
$\begin{array}{l}\text { Well-defined, with clear margins } \\
2 \text { to } 10 \mathrm{~mm} \text { in size in at least one plane }\end{array}$ & At least half surrounded by brain parenchyma \\
$\begin{array}{l}\text { Distinct appearance from potential mimics (e.g., calcium and iron deposits, bone, or } \\
\text { vessel flow voids) }\end{array}$ & $\begin{array}{c}\text { The differentiation from a mimic may be uncertain, but a } \\
\text { Not part of an intraparenchymal hemorrhage or contusion }\end{array}$ \\
\hline
\end{tabular}

To be classified as a definite microbleed, all of the criteria in the left column had to be met. Configurations that were less hypo-intense, less welldefined, and/or differentiable with less certainty from a potential mimic were classified as a possible microbleed (right column)

Table 2 Imaging parameters

\begin{tabular}{lll}
\hline & MPRAGE & SWI \\
\hline TR (ms) & 2300 & 27 \\
TE (ms) & 2.98 & 20.0 \\
TI (ms) & 900 & \\
Flip angle (degrees) & 9 & 15 \\
Bandwidth (Hz/pixel) & 240 & 120 \\
Slice thickness (mm) & 1.0 & 1.0 \\
Voxel-size (mm) & $1.0 \times 1.0 \times 1.0$ & $1.0 \times 1.0 \times 1.0$ \\
FOV (mm) & 256 & 250 \\
TA (minutes) & $5: 21$ & $7: 44$ \\
Dimension & $3 \mathrm{D}$ & $3 \mathrm{D}$ \\
\hline
\end{tabular}

Adapted from [9]

$T A$ acquisition time

\section{Post-comparison findings}

The SWI images with the segmentations at $t 1$ and $t 2$ were registered using the linear registration tool FLIRT [21, 22 ], and the segmentations were automatically compared between the time-points. In order to improve the quality of our microbleed evaluation, we used classification differences between the time-points to select microbleeds deserving a second look. AE and BG re-evaluated each of these microbleeds, based on the configuration per scan, not taking into account temporal pathophysiological assumptions. In case of equivocality, the original classification was maintained. In the rest of the paper, when referring to post-comparison findings, we refer to our classification at this stage.

\section{Causes of differences}

Each microbleed with a different single-scan classification between the time-points was reviewed by AE and BG to identify the cause of difference, selecting one of the options listed in Table 4, including "pathophysiological difference." The latter was only used if, when comparing the SWI scans, it was highly likely that the microbleed pathophysiologically changed from the first to the second scan.

To maximize the quality of the classification, we reviewed each microbleed with a possible MRI technical or pathophysiological difference (i.e., those classified as (possible) pathophysiological or artificial cause of difference) with an MR physicist (MM, 11 years of experience), incorporating additional MR sequences, if required (FLAIR, T1, T2, DWI).

\section{Blinding}

In order to avoid observer bias due to influence of clinical information on the classification of candidate microbleeds, the neuroradiologists and MR physicist interpreting the SWI scans (AE, BG, MM) had no access to clinical information.

\section{Statistical analyses}

Data were analyzed using SPSS 25 and STATA 15 statistical software. We performed Kolmogorov-Smirnov test to test normality of the distribution of the number of microbleeds per patient. As they were not normally distributed, we performed Wilcoxon signed-rank tests to compare numbers of microbleeds between the time-points. Within the subset of single-scan possible microbleeds at $t 1$, we aimed to identify the most relevant predictors of the classification at $t 2$. To identify predictors of the classification at $t 2$, we performed two-level multinomial logistic regression with random effects for patient ID. Patient-level variables were age (years), gender, GCS at arrival at the emergency department, signs of elevated intracranial pressure at the emergency department (yes/no), normalized extra-axial injury score, number of intraparenchymal hemorrhages, number of non-hemorrhagic contusions, and total number of definite microbleeds at $t 1$ (single-scan). Microbleed level variables were shape (elongated versus spherical), distance from the inner surface of the skull (mm), and number of definite microbleeds at $t 1$ (singlescan) within $2 \mathrm{~cm}$ of the microbleed under evaluation. The dependent variable in these analyses was the classification at 

and concomitant lesions in each MARS-region 3 weeks after TBI
Table 3 Number of microbleeds

\begin{tabular}{llllllll}
\hline MARS-region & Definite microbleeds & Possible microbleeds & IPH & NHC & SAH & SDH & EDH \\
\hline Frontal & $314.0(6.9)$ & $56.7(1.0)$ & $22(0)$ & $0(0)$ & $5(0)$ & $0(0)$ & $0(0)$ \\
Temporal & $246.8(7.8)$ & $55.6(.8)$ & $20(0)$ & $2(0)$ & $1(0)$ & $0(0)$ & $1(0)$ \\
Parietal & $75.8(1.8)$ & $22.3(.0)$ & $2(0)$ & $0(0)$ & $7(0)$ & $0(0)$ & $0(0)$ \\
Cerebellum & $19.2(.0)$ & $10.3(.0)$ & $1(0)$ & $0(0)$ & $16(0)$ & $0(0)$ & $0(0)$ \\
Brainstem & $33.6(.2)$ & $5.3(.0)$ & $1(0)$ & $2(0)$ & $0(0)$ & $1(0)$ & $0(0)$ \\
Optic pathways & $16.7(.0)$ & $5.1(.0)$ & $0(0)$ & $0(0)$ & $0(0)$ & $0(0)$ & $0(0)$ \\
Corpus callosum & $39.9(.0)$ & $4.9(.0)$ & $3(0)$ & $1(0)$ & $0(0)$ & $0(0)$ & $0(0)$ \\
Insula & $10.2(.0)$ & $4.6(.0)$ & $1(0)$ & $0(0)$ & $0(0)$ & $0(0)$ & $0(0)$ \\
External capsule & $13.1(.0)$ & $3.9(.0)$ & $0(0)$ & $0(0)$ & - & - & - \\
Occipital & $32.2(.8)$ & $3.1(.0)$ & $2(0)$ & $0(0)$ & $1(0)$ & $0(0)$ & $1(0)$ \\
Internal capsule & $9.4(.0)$ & $2.1(.0)$ & $1(0)$ & $0(0)$ & - & - & - \\
Basal ganglia & $25.7(.0)$ & $2.0(.0)$ & $2(0)$ & $0(0)$ & - & - & - \\
Deep and perive- & $24.9(.2)$ & $1.5(.0)$ & $1(0)$ & $0(0)$ & - & - & - \\
$\quad$ ntricular white & & & & & & & \\
$\quad$ matter & & & & & & & - \\
Thalamus & $4.5(.0)$ & $173(5)$ & $0(0)$ & $0(0)$ & - & - & - \\
Whole brain & $865(24)$ & $56(1)$ & $5(0)$ & $30(1)$ & $1(0)$ & $2(0)$ \\
\hline
\end{tabular}

Values are total number of lesions (median number of lesions per patient) at $t 1$ at single-scan evaluation. Only lesions that met the criteria described in the Online Resource were counted; e.g., epidural hematomas of $<1-\mathrm{cm}$ thickness were excluded from these numbers. No values regarding extra-axial hemorrhage are presented for the regions "deep and periventricular white matter," "basal ganglia," "thalamus," "internal capsule," and "external capsule," as due to their location they cannot be adjacent to extra-axial injury

The anatomical MARS regions are presented in descending order of number of possible microbleeds at $t 1$ MARS Microbleed Anatomical Rating Scale [13], TBI traumatic brain injury, IPH intraparenchymal hemorrhages, $N H C$ non-hemorrhagic contusions, $S A H$ subarachnoid hemorrhages, $S D H$ subdural hematomas, $E D H$ epidural hematomas

$t 2$, using staying possible as the reference category. The variables with $p<0.2$ in the univariable analyses were included in multivariable analysis. Spearman $r$ between the independent variables was $<0.3$ for each pair of variables. Microbleeds in the MARS region's deep and periventricular white matter, basal ganglia, thalamus, and internal and external capsule were excluded from the analyses on extra-axial injury score, as their extra-axial injury score is zero by definition. Because

Table 4 Causes of classification differences between $t 1$ and $t 2$

\begin{tabular}{|c|c|c|c|}
\hline \multirow[t]{2}{*}{ Etiological category } & \multirow[t]{2}{*}{ Subcategory } & \multicolumn{2}{|c|}{ Number of microbleeds (\%) } \\
\hline & & $\begin{array}{l}\text { From possible to } \\
\text { no }(n=93)\end{array}$ & $\begin{array}{l}\text { From possible } \\
\text { to definite }(n=27)\end{array}$ \\
\hline Pathophysiological difference ${ }^{a}$ & & $7(8 \%)$ & $5(19 \%)$ \\
\hline Possible pathophysiological difference ${ }^{\mathrm{b}}$ & & $2(2 \%)$ & $5(19 \%)$ \\
\hline \multirow[t]{3}{*}{ Interpretation-related } & Equivocal classification of microbleed ${ }^{\mathrm{c}}$ & $4(4 \%)$ & $1(4 \%)$ \\
\hline & Missed by CAD and expert & $0(0 \%)$ & $0(0 \%)$ \\
\hline & Misinterpreted & $39(42 \%)$ & $5(19 \%)$ \\
\hline \multirow[t]{3}{*}{ Technique-related } & Misregistration or missegmentation & $2(2 \%)$ & $7(26 \%)$ \\
\hline & Artifact hampering the interpretation ${ }^{\mathrm{d}}$ & $36(39 \%)$ & $4(15 \%)$ \\
\hline & Artifact precluding evaluation ${ }^{\mathrm{e}}$ & $3(3 \%)$ & $0(0 \%)$ \\
\hline
\end{tabular}

${ }^{a}$ When comparing the susceptibility weighted imaging (SWI) scans, it is highly likely that the microbleed pathophysiologically changed from the first to the second scan

${ }^{\mathrm{b}}$ The microbleed looks different at $t 1$ and $t 2$, but uncertainty remains whether the different appearance reflects a pathophysiological or a technical difference

${ }^{c}$ Both possible and definite microbleed can be advocated

${ }^{\mathrm{d}}$ Visible at both scans, but classified differently due to an artifact. For example, microbleed's appearance changes from definite to possible due to blurring by an artifact

${ }^{\mathrm{e}}$ Artifact making the microbleed invisible at one scan while visible at the other 
of the nominal nature of the variable "MARS region," we separately evaluated differences in the classification at $t 2$ between anatomical locations, using Kruskal-Wallis test with Bonferroni-corrected post hoc pairwise comparisons. Values are expressed as median (interquartile range) unless otherwise stated. Differences with $p<0.05$ were considered significant.

\section{Results}

A total of 1038 non-contiguous microbleeds were detected, 173 of which were possible microbleeds (Table 3 ).

\section{Longitudinal evolution of possible microbleeds}

Ninety-three of the 173 (53.8\%) possible microbleeds at $t 1$ corresponded to no microbleed at $t 2,53(30.6 \%)$ to a possible, and $27(15.6 \%)$ to a definite microbleed.

\section{Predictors of the longitudinal evolution of possible microbleeds}

In univariable but not in multivariable analyses, the odds of corresponding to no microbleed at $t 2$ were higher in males (Table 5).

Both in univariable and in multivariable analyses, the odds of corresponding to a definite microbleed at $t 2$ were higher for microbleeds in a MARS-region with a higher

Table.5 Predictors of the $t 2$-classification of possible microbleeds at $t 1$

\begin{tabular}{|c|c|c|c|c|}
\hline & \multicolumn{2}{|c|}{$\begin{array}{l}\text { OR ( } 95 \% \text { CI, } p) \text { of classification as "no micro- } \\
\text { bleed" at } t 2\end{array}$} & \multicolumn{2}{|c|}{$\begin{array}{l}\text { OR ( } 95 \% \text { CI, } p \text { ) of classification as "definite micro- } \\
\text { bleed" at } t 2\end{array}$} \\
\hline & Univariable analyses & Multivariable analysis & Univariable analyses & Multivariable analysis \\
\hline Elongated shape $^{a}$ & $.56(.17-1.87, .350)$ & - & $1.11(.24-5.17, .892)$ & - \\
\hline $\begin{array}{l}\text { Distance from inner surface of skull } \\
(\mathrm{mm})\end{array}$ & $1.02(.97-1.08, .368)$ & - & $1.02(.96-1.09, .527)$ & - \\
\hline $\begin{array}{l}\text { Number of definite microbleeds } \\
\text { within } 2 \mathrm{~cm} \text { of the microbleed } \\
\text { under evaluation }^{b}\end{array}$ & $.97(.83-1.12, .638)$ & $.95(.80-1.13, .550)$ & $1.23(1.04-1.45, .015)^{*}$ & $1.22(1.02-1.45, .028)^{*}$ \\
\hline Extra-axial injury score ${ }^{c}$ & $1.07(.77-1.50, .680)$ & $1.34(.89-2.03, .159)$ & $1.54(1.03-2.29, .034)^{*}$ & $1.77(1.09-2.85, .020)^{*}$ \\
\hline $\begin{array}{l}\text { Number of intraparenchymal } \\
\text { hemorrhages }\end{array}$ & $.99(.42-2.35, .987)$ & - & $.56(.11-2.83, .484)$ & - \\
\hline $\begin{array}{l}\text { Number of non-hemorrhagic } \\
\text { contusions } s^{c}\end{array}$ & $.41(.07-2.28, .307)$ & - & $-{ }^{f}$ & - \\
\hline $\begin{array}{l}\text { Total number of definite micro- } \\
\text { bleeds at } t 1^{\mathrm{b}}\end{array}$ & $1.00(.99-1.02, .671)$ & - & $1.01(.98-1.03, .547)$ & - \\
\hline GCS at arrival at the ED & $.90(.74-1.10, .300)$ & $.90(.74-1.10, .319)$ & $1.20(.98-1.47, .072)^{(*)}$ & $1.19(.99-1.43, .071)$ \\
\hline Male gender & $2.90(1.10-7.68, .032)^{*}$ & $3.08(.98-9.68, .054)$ & $1.43(.35-5.81, .619)$ & $1.22(.33-4.46, .765)$ \\
\hline Age $(\text { years })^{\mathrm{d}}$ & $.99(.96-1.02, .519)$ & - & $.97(.93-1.02, .221)$ & - \\
\hline $\begin{array}{l}\text { Signs of elevated intracranial pres- } \\
\text { sure at the ED }\end{array}$ & $.84(.45-1.58, .591)$ & - & .53 (no convergence achieved) & - \\
\hline
\end{tabular}

Results of two-level multinomial logistic regression analyses with random effects for patient ID, using the classification at $t 2$ as the dependent variable. Staying possible is the reference category. The multivariable analyses include all independent variables with $p<.2$ in the univariable analyses (see Online Resource for a list of the independent variables). For example, for each additional definite microbleed within $2 \mathrm{~cm}$ of the possible microbleed under evaluation, the odds of being classified as a definite microbleed at $t 2$ independently increased with a factor 1.22

${ }^{*} p<.05$

${ }^{(*)}$ included in multivariable analysis, because $p<.2$ in univariable analysis

a Defined as longest axis $\geq 2 *$ short axis

${ }^{\mathrm{b}}$ At single-scan evaluation

${ }^{\mathrm{c}}$ In the same MARS region at $t 1$, normalized to the volume of the MARS region in MNI space

${ }^{\mathrm{d}}$ At the day of trauma

${ }^{\text {e}}$ Generalized or hemispheric edema with effacement of sulci, compressed ventricles or basal cisterns and midline shift $>5 \mathrm{~mm}$ (yes/no)

${ }^{\mathrm{f}}$ None of the possible microbleeds at $t 1$ in a MARS region with non-hemorragic contusion, corresponded to a definite microbleed at $t 2$

GCS Glasgow Coma Scale score, ED emergency department 
extra-axial injury score, and for microbleeds with a larger number of definite microbleeds within $2 \mathrm{~cm}$ of the microbleed under evaluation (Table 5 ).

Figure 2 shows the anatomical distribution of possible microbleeds at $t 1$ corresponding to no, possible, and definite microbleeds at $t 2$. Overall, the classification at $t 2$ differed between the regions $(p<0.05)$. No difference between individual pairs of anatomical regions was demonstrated.

\section{Causes of differences}

Table 4 summarizes the frequency of the various causes of classification differences. Figure 3 illustrates each etiological category.

\section{From possible to no microbleed}

The classification difference of 78 of the 93 possible microbleeds corresponding to no microbleed at $t 2$ was attributable to misinterpretation or to an artifact (84\%, Table 4).

The artifacts hampering or precluding the interpretation were due to susceptibility at an air-tissue interface (16), pulsation (14), motion (8), and susceptibility induced by extraaxial blood (1). The misinterpretations reflected a vessel (26), subarachnoid blood (8), subarachnoid blood or a vessel (3), metal (1), and an unclassified artifact (1) that were erroneously taken for a possible microbleed. Five of the pathophysiologically changed microbleeds had decreased in diameter, while 2 had completely disappeared.

\section{From possible to definite microbleed}

The classification difference of 22 of the 27 possible microbleeds corresponding to a definite microbleed at $t 2$ was attributable to a pathophysiological or possible pathophysiological difference, to misregistration/missegmentation or to misinterpretation ( $81 \%$, Table 4). All of the 5 pathophysiologically changed microbleeds had grown over time. The artifacts hampering the interpretation were due to pulsation (2), motion (1), and susceptibility at an air-tissue interface (1). The misinterpretations were a microbleed where a vessel (3) or subarachnoid blood (1) was erroneously considered to be in the differential diagnosis, and one misinterpretation could not be explained.

\section{Discussion}

We have shown that $16 \%$ of the possible microbleeds at baseline correspond to a definite microbleed at follow-up, while $54 \%$ correspond to no microbleed, and uncertainty remains in $31 \%$.

We evaluated possible microbleeds after TBI with 3-T SWI in a setting reflecting radiological practice, i.e., interpretation of possible microbleeds without any information on the findings at follow-up. Previous reports on possible microbleeds evaluate non-traumatic microbleeds assessed with GRE T2*-weighted 1.5-T MRI. Their objective differs

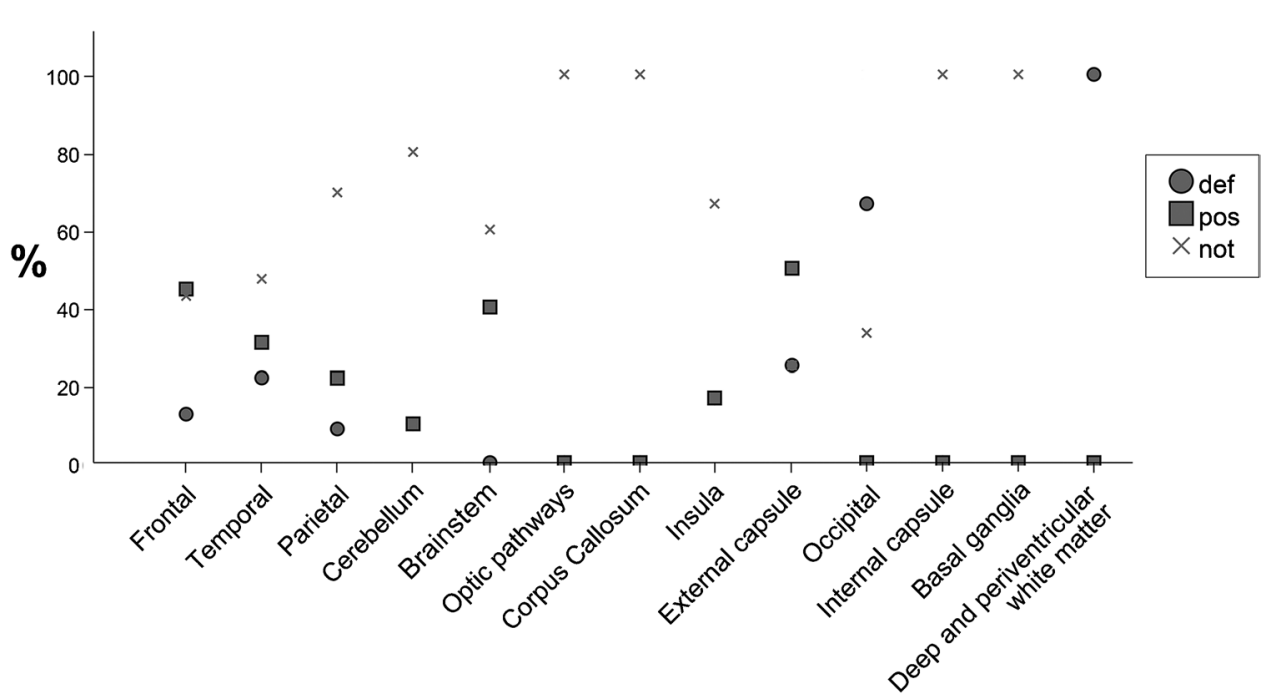

Fig. 2 Anatomical distribution of possible microbleeds at $t 1$ corresponding to no, possible, and definite microbleeds at $t 2$. The $\mathrm{x}$-axis shows the anatomical regions in descending order of number of possible microbleeds at $t 1$. Thalamus is not on the $\mathrm{x}$-axis, as it contained no possible microbleeds at $t 1$. The $\mathrm{y}$-axis shows per region the per- centage of possible microbleeds at $t 1$ that was classified as definite (circle), possible (square), or no (cross) microbleed at $t 2 . t 1: 3(2-5)$ weeks after TBI, $t 2: 26$ (25-28) weeks after TBI, def: definite microbleed, pos: possible microbleed, not: no microbleed 

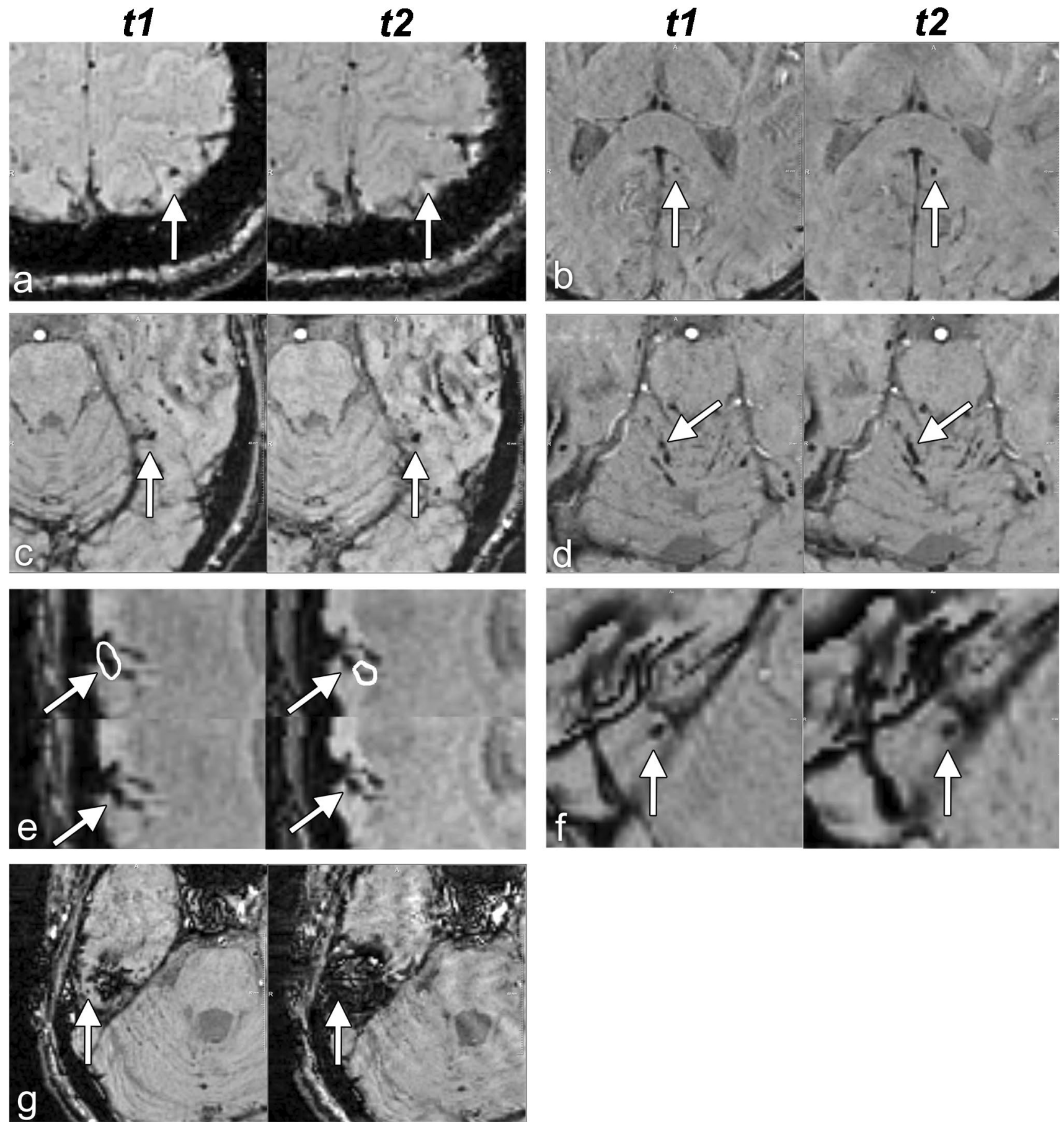

Fig. 3 Examples of possible microbleeds at $t 1$ classified differently at t2. a Pathophysiological difference (disappearance); b possible pathophysiological difference, with slightly decreased signal intensity and increased blooming at $t 2$, which may be caused by a technical difference between the scans or by a pathophysiological change; $\mathbf{c}$ equivocal classification, especially at $t 1$ this configuration can as well be classified as a possible microbleed as as a continuation of the blood vessel medial to it; $\mathbf{d}$ misinterpreted otherwise: this subarachnoid blood was mistaken for a possible microbleed at $t 1$; e misregistration

or missegmentation: at both time-points, this possible microbleed was segmented too small; the segmentations did not overlap, resulting in a false mismatch in the automatic comparison step; $\mathbf{f}$ artifact hampering the interpretation (susceptibility at air-tissue interface); $\mathbf{g}$ artifact precluding evaluation at $t 2$ (susceptibility at air-tissue interface). Images are axial images. Arrows point to the microbleeds discussed. In e, the automatic segmentation resulted in the closed curves. $t 1: 3(2-5)$ weeks after TBI, $t 2$ : 26 (25-28) weeks after TBI 
from ours: they focus on the interobserver agreement at a single time-point, while we evaluate the temporal evolution of possible microbleeds [13, 14]. Whereas previous studies report patient-based numbers and include a total of 24-46 [14] and 52-63 [13] possible microbleeds, we report on 173 individual possible microbleeds, allowing evaluation of predictors of the longitudinal evolution of possible microbleeds.

\section{Predictors of the longitudinal evolution of possible microbleeds}

The predictors of the longitudinal evolution of possible microbleeds comprise anatomical, demographic, and injuryrelated variables. The associations probably rely on a combination of interpretational and pathophysiological factors.

Only the minority of possible microbleeds evolve into a definite microbleed. This happens most frequently to possible microbleeds surrounded by many definite microbleeds. This may be due to an unfavorable micro-environment, caused by an inflammatory response to blood-derived substances in the perifocal zone, especially free iron and heme [23]. In this setting, a minimal amount of blood products, having oozed into the parenchyma at $t 1$, barely visible on SWI and thus classified as a possible microbleed, could progress into a definite microbleed at $t 2$. While in a favorable micro-environment, this minimal amount of blood products could have been resorbed. Also, a higher extra-axial injury load is independently predictive of turning into a definite microbleed. This may be related to a loss of compression over time, as extra-axial injury mostly decreases from baseline to follow-up. However, also the reliability of SWI interpretation may improve with a reduction of extra-axial injury over time, thus reducing the necessity of classifying a microbleed as possible. Alternatively, the etiology of a possible microbleed near many definite microbleeds or near extra-axial injury might differ from other microbleeds.

Half of the possible microbleeds correspond to no microbleed at follow-up. This may reflect resorption of minimal amounts of blood products in some cases, but may very well reflect the intrinsic uncertainty of the classification "possible" in the majority [24]. Though possible microbleeds in males have increased odds of disappearing, this relation is not causal, as in multivariable analyses this relation is not significant. In the optic pathways, internal capsule, basal ganglia, and corpus callosum, all of the possible microbleeds disappeared at $t 2$. However, the numbers are too low to draw conclusions about the relation between anatomical location and the course of possible microbleeds.

As the sparsity of statistical differences may have been caused by the limited number of possible microbleeds per region and per candidate predictor, we also discuss possible inferences from trends. First, we discuss trends appearing from the regression analyses (Table 5), and then we zoom in to the anatomical regions (Fig. 2 and Table 3 ).

Whereas a higher extra-axial injury load is independently predictive of turning into a definite microbleed, there is also a trend of a higher extra-axial injury load being predictive of turning into no microbleed (Table 5). This paradox indicates that in regions with more extra-axial injury, fewer possible microbleeds stay possible. This suggests that the role of extra-axial injury may not be a pathophysiological influence on microbleeds, but rather an influence on the reliability of microbleed evaluation. Indeed, the reliability of SWI interpretation may improve with the resorption of extra-axial injury over time, thus reducing the necessity of classifying a microbleed as possible: the criteria for classifying it as a definite microbleed or as no microbleed are more easily met in the absence of interfering concomitant extra-axial lesions.

We limit our discussion of the location of possible microbleeds to the regions with $>5$ possible microbleeds, i.e., frontal, temporal, parietal, and cerebellum. Mainly due to their different locations in relation to the skull and the dural folds, the predominant mechanisms of injury differ between these regions, with the frontal and temporal lobe being more similar than the parietal lobe and cerebellum. Also the course of possible microbleeds is similar in the frontal and temporal lobes, and different in the parietal lobe and cerebellum (Fig. 2). On the one hand, this may suggest pathophysiological differences between microbleeds at the different locations. On the other hand, possible pathophysiological differences of the microbleeds themselves cannot be unraveled from the role of concomitant injury, which is unevenly distributed over the regions (Table 3). The cerebellum and parietal region are the regions with the largest proportion of microbleeds being classified as possible. In line with the discussion above, these same regions are the regions with the highest subarachnoid hemorrhage load (Table 3), which may have caused the proportion of microbleeds being classified as possible in these regions to be high. Indeed, in these two regions, the vast majority of possible microbleeds turned into no microbleed at follow-up, whereas in the regions with less subarachnoid hemorrhage, i.e., the temporal and to a lesser extent the frontal region, a substantial proportion of the possible microbleeds turned into a definite microbleed at follow-up (Fig. 2). This suggests that the presence of concomitant subarachnoid hemorrhage renders the interpretation of microbleeds more uncertain, but that the uncertainty fades when the subarachnoid blood is resorbed. Intraparenchymous hemorrhage on the other hand may be associated with remaining uncertainty on the classification of microbleeds, even after evolution of the intraparenchymous hematoma: the vast majority of intraparenchymous hemorrhages was observed in the frontal and temporal lobe (Table 3), where a substantial proportion of possible microbleeds stayed possible at follow-up (Fig. 2). In conclusion, a follow-up SWI scan could be advocated especially for 
possible microbleeds located in regions with subarachnoid hemorrhage, and the yield of a follow-up scan may be lower for possible microbleeds located in regions with intraparenchymous hemorrhage.

\section{Recommendations for radiological practice}

In radiological practice, possible microbleeds are encountered on a daily basis. Though for the majority of patients the interpretation of possible microbleeds will not change management, prognostication, or trauma grading, for some patients it will. The accuracy of the interpretation of possible microbleeds is especially important, if they affect the grading of traumatic/diffuse axonal injury, or if they are in patients with few or no definite microbleeds, especially in forensic cases, or in patients with persistent post-traumatic symptoms to which the contribution of traumatic and nontraumatic factors need to be disentangled.

This is a first step in the answer to the question of how to deal with a possible microbleed on a single SWI scan in a clinical radiological setting. On the one hand, only $16 \%$ of the possible microbleeds at $t 1$ turns into a definite microbleed, and therefore possible microbleeds may be regarded as no microbleed. On the other hand, uncertainty remains in $31 \%$ : on two separate scans they are classified as a possible microbleed. As adding a second scan did result into a more certain classification (definite or no microbleed) of $69 \%$ of the possible microbleeds on a single scan, a second SWI scan could be advocated in case of clinical consequences. However, even though classified by two neuroradiologists, as many as $25 \%$ of the possible microbleeds were misinterpreted at single scan evaluation. In addition to the intrinsic difficulty of the evaluation of possible microbleeds, this may also be due to the large total amount of microbleeds and false-positive CAD detections to be interpreted (we misinterpreted $4 \%$ of all of the possible and definite microbleeds, and circa $1.8 \%$ of all of the locations that were detected by the CAD system (denominator inferred from Van den Heuvel et al. [9])). Therefore, before performing a followup scan, possible microbleeds merit a second inspection to make sure that they cannot be reclassified as a definite or no microbleed: the cause of classification difference between the timepoints is misinterpretation in as many as $25 \%$ of the possible microbleeds. Alternatively, the decision on an individual possible microbleed could incline toward a microbleed if it is near many other microbleeds or in a region with extra-axial injury.

\section{Limitations}

Of the screened patients, only a small proportion was finally included in the study. This was mainly due to lack of informed consent or decease before the first MRI, reflecting the difficulty of patient inclusion from the ICU. Of the remaining patients, 8 had to be excluded due to motion artifacts, a well-known challenge of performing MRI in patients with severe TBI. Both effects may have introduced a selection bias toward a lower injury severity, possibly with lower microbleed loads.

A single neuroradiologist evaluated a substantial proportion of the microbleeds. Aware of the resulting vulnerability to inter-rater variability-based bias, the second neuroradiologist was consulted in case of the slightest doubt. Additionally, the second neuroradiologist reviewed a random sample of 30 of the other microbleeds and agreed on all of them. The automatic comparison between the time-points could be viewed as a surrogate co-reader, prompting to re-evaluate each differing microbleed in consensus meetings.

Another limitation is that at the single-scan stage, we only evaluated SWI. In the post-comparison stage, we used additional sequences. We had no access to the SWI-phase images ${ }^{7}$.

The lack of histopathologic correlation limits the interpretation of the longitudinal findings. Ten percent of the possible microbleeds showed evidence of a pathophysiological change over time, but they were too few to draw conclusions on predictors of their evolution. As no repeat SWI scan at $t 1$ was available, the effects of technique (re-test reliability) cannot be separated from the physical longitudinal evolution in the search for predictors of the classification at follow-up. This is a purely diagnostic study. The number of patients is insufficient to perform meaningful patient-wise analyses and to evaluate the clinical significance of our findings. Though the data acquisition on a single scanner improved the data homogeneity, it limits the generalizability of our findings to clinical practice.

\section{Conclusion}

Sixty-nine percent of the possible microbleeds at baseline are classified differently at follow-up. Only $16 \%$ of them represent a definite microbleed at follow-up.

The longitudinal evolution differs between locations and micro-environments. Closeness to extra-axial injury and closeness to definite microbleeds are independently predictive of corresponding to a definite microbleed at follow-up. The reclassification level differs between anatomical locations, though no difference between individual pairs of anatomical regions was demonstrated. Ten percent of the classification differences between baseline and follow-up reflect a pathophysiological change.

Future research is needed to evaluate the pathophysiological and interpretational backgrounds of our findings.

Our results support disregarding possible microbleeds in the absence of clinical consequences. In case of clinical 
consequences, however, a follow-up SWI scan could be obtained to exclude evolution into a definite microbleed, especially if the possible microbleed is surrounded by many definite microbleeds, or is in a region with extraaxial injury.

Supplementary Information The online version contains supplementary material available at https://doi.org/10.1007/s00234-021-02839-z.

Acknowledgements We would like to thank T.M.J. Andriessen, psychologist at Saffier, Den Haag for her contribution to the collection of data. We would like to thank F.L. van der Panne, medical photographer at Erasmus Medical Center, and K. Koschmieder, PhD student traumatic imaging analysis at Radboud University Medical Center, for their help with figure design. We would also like to thank the patients who participated in this study. We would like to thank Trauma Regio Oost, Radboud University Medical Center, for partly funding this investigator-initiated study.

Author contribution Van der Eerden, Van den Heuvel, Vos, Platel, Góraj, and Manniesing contributed to the study conception and design. Material preparation, data collection, and analysis were performed by all authors. The first draft of the manuscript was written by Van der Eerden, Platel, Góraj, and Manniesing, and all authors commented on previous versions of the manuscript. All authors read and approved the final manuscript.

Funding This is an investigator-initiated study, partly funded by Trauma Regio Oost, Radboudumc, Nijmegen. The funding organization did not participate in the design and conduct of the study; collection, management, analysis, and interpretation of the data; preparation, review, and approval of the manuscript; and the decision to submit the manuscript for publication.

Data availability Upon request.

Code availability Upon request.

\section{Declarations}

Conflict of interest The authors have no financial or non-financial interests to disclose.

Ethics approval Ethical approval was obtained from Radboudumc's Institutional Review Board.

The study was performed in accordance with the ethical standards as laid down in the 1964 Declaration of Helsinki and its later amendments.

The same patients were reported in another manuscript [9]. In that report, we evaluated the correlation between definite cerebral microbleeds and white matter integrity. The current study focuses on possible microbleeds. To avoid self-plagiarism, we refer to the other manuscript in case of equal methodology or patient descriptions.

Consent to participate and consent for publication All patients or their next of kin gave written informed consent.

Open Access This article is licensed under a Creative Commons Attribution 4.0 International License, which permits use, sharing, adaptation, distribution and reproduction in any medium or format, as long as you give appropriate credit to the original author(s) and the source, provide a link to the Creative Commons licence, and indicate if changes were made. The images or other third party material in this article are included in the article's Creative Commons licence, unless indicated otherwise in a credit line to the material. If material is not included in the article's Creative Commons licence and your intended use is not permitted by statutory regulation or exceeds the permitted use, you will need to obtain permission directly from the copyright holder. To view a copy of this licence, visit http://creativecommons.org/licenses/by/4.0/.

\section{References}

1. Nguyen R, Fiest KM, McChesney J et al (2016) The international incidence of traumatic brain injury: a systematic review and meta-analysis. Can J Neurol Sci 43(6):774-785

2. Peeters W, van den Brande R, Polinder S et al (2015) Epidemiology of traumatic brain injury in Europe. Acta Neurochir (Wien) 157(10):1683-1696

3. Griffin AD, Turtzo LC, Parikh GY et al (2019) Traumatic microbleeds suggest vascular injury and predict disability in traumatic brain injury. Brain 142(11):3550-3564

4. Geurts BH, Andriessen TM, Goraj BM, Vos PE (2012) The reliability of magnetic resonance imaging in traumatic brain injury lesion detection. Brain Inj 26(12):1439-1450

5. Greenberg SM, Vernooij MW, Cordonnier C et al (2009) Cerebral microbleeds: a guide to detection and interpretation. Microbleed Study Group. Lancet Neurol 8(2):165-174

6. Izzy S, Mazwi NL, Martinez S et al (2017) Revisiting grade 3 diffuse axonal injury: not all brainstem microbleeds are prognostically equal. Neurocrit Care 27(2):199-207

7. Haller S, Vernooij MW, Kuijer JPA, Larsson EM, Jäger HR, Barkhof F (2018) Cerebral microbleeds: imaging and clinical significance. Radiology 287(1):11-28

8. Liu J, Kou Z, Tian Y (2014) Diffuse axonal injury after traumatic cerebral microbleeds: an evaluation of imaging techniques. Neural Regen Res 9(12):1222-1230

9. Van der Eerden AW, van den Heuvel TLA, Perlbarg V et al (2021) Traumatic cerebral microbleeds in the subacute phase are practical and early predictors of abnormality of the normalappearing white matter in the chronic phase. AJNR Am J Neuroradiol 42(5):861-867

10. Haghbayan H, Boutin A, Laflamme M et al (2017) The prognostic value of MRI in moderate and severe traumatic brain injury: a systematic review and meta-analysis. Crit Care Med 45(12):e1280-e1288

11. Galanaud D, Perlbarg V, Gupta R et al (2012) Assessment of white matter injury and outcome in severe brain trauma: a prospective multicenter cohort. Anesthesiology 117(6):1300-1310

12. Wallace EJ, Mathias JL, Ward L (2018) The relationship between diffusion tensor imaging findings and cognitive outcomes following adult traumatic brain injury: a meta-analysis. Neurosci Biobehav Rev 92:93-103

13. Gregoire SM, Chaudhary UJ, Brown MM et al (2009) The Microbleed Anatomical Rating Scale (MARS): reliability of a tool to map brain microbleeds. Neurology 21:1759-1766

14. Cordonnier C, Potter GM, Jackson CA et al (2009) Improving interrater agreement about brain microbleeds: development of the Brain Observer MicroBleed Scale (BOMBS). Stroke 40(1):94-99

15. Wardlaw JM, Smith EE, Biessels GJ et al (2013) Neuroimaging standards for research into small vessel disease and its contribution to ageing and neurodegeneration. STandards for ReportIng Vascular changes on nEuroimaging (STRIVE v1). Lancet Neurol 12(8):822-838 
16. Ricciardi MC, Bokkers RP, Butman JA et al (2016) Trauma-specific brain abnormalities in suspected mild traumatic brain injury patients identified in the first 48 hours after injury: a blinded magnetic resonance imaging comparative study including suspected acute minor stroke patients. J Neurotrauma 34:23-30

17. MNI ICBM 152 non-linear 6th generation symmetric average brain stereotaxic registration model. http://nist.mni.mcgill. ca/?p=858 Accessed Dec 2017

18. Van den Heuvel TL, van der Eerden AW, Manniesing R et al (2016) Automated detection of cerebral microbleeds in patients with traumatic brain injury. Neuroimage Clin 12:241-251

19. FSL FNIRT. https://fsl.fmrib.ox.ac.uk/fsl/fslwiki/FNIRT Accessed Dec 2017

20. Jenkinson M, Beckmann CF, Behrens TE, Woolrich MW, Smith SM (2012) FSL Neuroimage 62(2):782-790
21. FSL FLIRT. https://fsl.fmrib.ox.ac.uk/fsl/fslwiki/FLIRT Accessed Dec 2017

22. Greve DN, Fischl B (2009) Accurate and robust brain image alignment using boundary-based registration. Neuroimage 48(1):63-72

23. Righy C, Bozza MT, Oliveira MF, Bozza FA (2016) Molecular, cellular and clinical aspects of intracerebral hemorrhage: are the enemies within? Curr Neuropharmacol 14(4):392-402

24. Liu W, Soderlund K, Senseney JS et al (2016) Imaging Cerebral Microhemorrhages in Military Service Members with Chronic Traumatic Brain Injury. Radiology 278(2):536-545

Publisher's note Springer Nature remains neutral with regard to jurisdictional claims in published maps and institutional affiliations. 